\title{
LARGE PENETRATION OF DISTRIBUTED PRODUCTIONS: DYNAMIC LINE RATING AND FLEXIBLE GENERATION, A MUST REGARDING INVESTMENT STRATEGY AND NETWORK RELIABILITY.
}

\author{
Peter Schell \& Bertrand Godard \\ Ampacimon-Belgium \\ peter.schell@ampacimon.com
}

Prof. Jean-Louis Lilien \& Huu-Minh Nguyen

University of Liège - Belgium

lilien@montefiore.ulg.ac.be

\author{
Vanessa De Wilde \\ Elia-Belgium \\ Vanessa.dewilde@elia.be
}

\author{
Olgan Durieux \\ ORES - Belgium \\ olgan.durieux@ores.net \\ Jean-Jacques Lambin \\ Elia-Belgium \\ jean-jacques.lambin@elia.be
}

\begin{abstract}
Dynamic Line Rating (DLR), a.k.a determining the maximum rating of overhead lines based on real-time weather parameters has the potential to significantly increase the power transport \& distribution capacity of the grid in favorable weather conditions. Combining active network management (curtailable generation) with DLR as trigger offers a solution out-there for the system operator that combines technical feasibility, adequate implementation timing and economic viability. Flexible generation being already in use on the ELIA network, the potential benefits of using it in conjunction with DLR will be analyzed.
\end{abstract}

This paper will give a first answer to the following questions:

Q1: What is the influence of a system of active network management using DLR on the investment strategy?

Q2: How is the reliability of the distribution system guaranteed in this new much more dynamic operation approach?

\section{INTRODUCTION}

Integrating large quantities of distributed generation in areas with limited connection capacity is a problem that grid system operators face more and more often.

Historically operational exploitation, for security reasons, was based on static line ratings. The static line rating is a conservative value based on the line's thermal limit calculated for an unlimited period of time and during unfavorable weather conditions. To relax somewhat this limit, that often leads to higher security margins than needed, seasonal ratings taking into account the medium ambient seasonal temperature are currently used.

As the capacity of overhead line changes according to weather conditions like wind speed and direction, air temperature, solar radiation, the calculation of real-time rating either using measured weather data or using dynamic line rating devices installed on the line can make additional capacity available. This additional capacity could be dynamically allocated to Distributed Generations (DG) through a system of active network management.
Based on this finding, Elia (the Belgian TSO) and ORES (one of the Belgian DSOs) decided to pilot this approach and evaluate its benefits, especially concerning the potential for generators to produce more energy without waiting for infrastructure reinforcements/extensions that often lead to both long delays and cumbersome costs.

To what extent can flexible generation and DLR influence investment strategy at the distribution level? And if they do to a large extent, how will we ensure that these innovative operation schemes do not negatively impact the reliability of the whole electricity distribution system? These are key questions that face all new "smart" system management approaches and DLR is no exception!

\section{DLR SMART SENSOR “AMPACIMON"}

Ampacimon $\odot$ is a new smart sensor available on the DLR market. It has a very original way of working. Directly attached on the conductor in the critical spans of the line (life line installation is possible), it outputs the sag of these spans without the need for any data, allowing for the error free certification of clearance in all circumstances! Fully autonomous, it is based on mechanical vibration observation; there is indeed a basic physical relationship that links sag to the measured vibration frequencies, see [1]. The reserve of sag, compared to its maximum value, is an image of the remaining capacity of the line. The key question is: what is the relationship between the wind power output at the wind farm location and the actual ampacity of the power line when the wind power is high (say over $60 \%$ of the nominal power)? In this pilot project, the wind farm was indeed very close to the power line, which was the most limiting factor for congestion purposes.

Such DLR sensors were already installed on $70 \mathrm{kV}, 150$ $\mathrm{kV}, 220 \mathrm{kV}$ and $400 \mathrm{kV}$ overhead lines to validate the technology. The results show that the technology works flawlessly and that substantial additional transmission capacity (=ampacity) are available. Fig. 1 shows the histogram of measured ampacity values (in relative value compared to static rating) for the months of Dec. 2011 and Jan. 2012 on a $70 \mathrm{kV}$ power line in the Ardennes. 


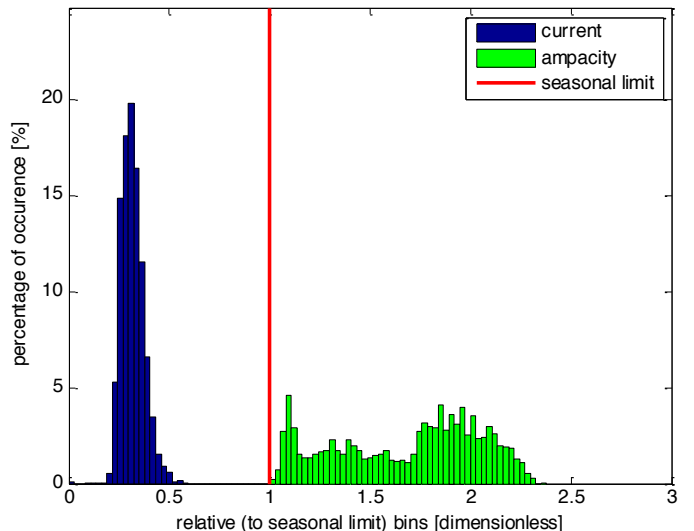

Fig. 1 Histogram of occurrences for dynamic rating (green) computed by "Ampacimon" against static rating (vertical line) on a $70 \mathrm{kV}$ transmission line in southern Belgium (dec 2011 - jan 2012).

(More information is available on www.ampacimon.com)

\section{CORRELATION BETWEEN WIND POWER OUTPUT AND AMPACITY}

There is no data available yet for the "Belgium east loop" region (our running pilot project with wind farms in the Ardennes) but based on data from another DLR installation near the Belgian coast in Brugge $(150 \mathrm{kV})$, Fig. 2, 3 and 4 show measurements which emphasize a clear relationship between wind farm production in the neighborhood and thermal rating of the line in a very similar arrangement as the "east loop".

Fig. 2 shows a one week period (in August 2010) with a strong correlation between available ampacity versus wind power produced some few $\mathrm{km}$ away from the power line site.

Fig. 3 and 4 show that in a very large percentage of the cases, substantial additional ampacity will be available when the wind farms are producing more than $60 \%$ of their rating (no potential congestion for lower values). Fig. 3 shows a scatter plot of the measured ampacities for all cases when Fig 4 is showing the same output but visualized using trends curves joining the mean value by power intervals of $5 \%$.

Wind speed on power line site being, in this case, strongly correlated to the wind speed at wind farm location, the production over $60 \%$ of the rated power is linked most of the time (but not always) to 1.8 times the static rating (extrapolated from Fig. 4 on the mean curve minus one standard deviation).

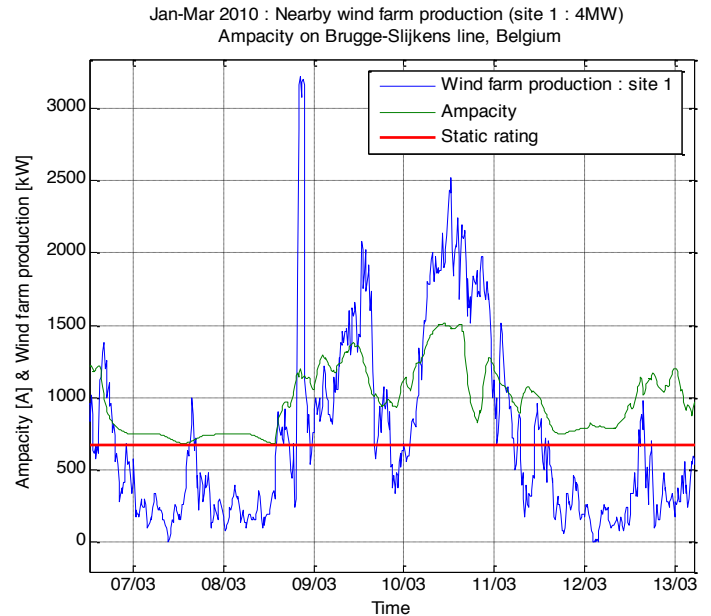

Fig. 2 An example of very good correlation between congested power line ampacity and wind power output on a nearby site. Extract from Brugge-Slijkens $150 \mathrm{kV}$ line near an offshore wind farm. 10 Ampacimon sensors have been installed since 2010.

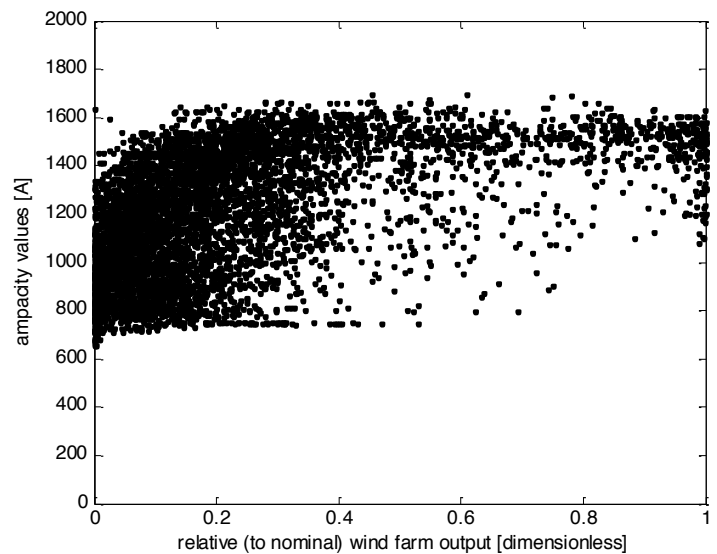

Fig. 3 Plot of Ampacity (y-axis) versus nearby wind power output (xaxis) for winter period Jan 2011-Mar 2011. $150 \mathrm{kV}$ Brugge-Slijkens line connected to a $21 \mathrm{MW}$ wind farm. (Static rating at $672 \mathrm{~A}$ )

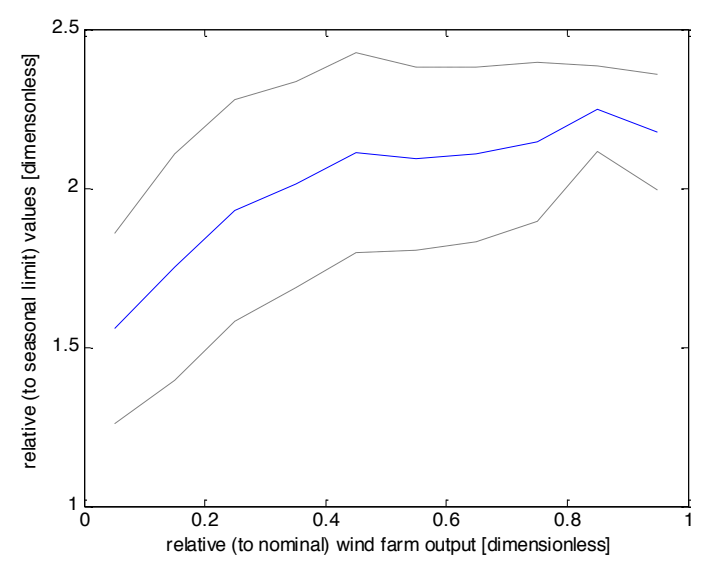

Fig. 4 Same data as for Fig. 3 limited to trends curve (with one standard deviation) and ordinates in relative value to static rating. 


\section{RUNNING PILOT PROJECT}

A former paper [2] has already been published for the CIRED 2011 conference about ANM (active network management) and actually running flexibility in wind generation based on power lines static rating. This current paper is more focused on the complementary use of DLR for the $70 \mathrm{kV}$-network -so-called "East Loop"located in the south-east of Belgium. (Fig. 6)

A high potential for distributed generation (mainly wind farms) exists in this area. The connection capacity to this part of the network is calculated classically for N-1 situations taking into account on one hand the installed power of DG and on the other hand the seasonal ratings of lines.

The percentage of time during which a wind farm generator injects its maximum power is relatively low: during $85 \%$ of the operation time, the produced power is below $50 \%$ of the rated maximum power (fig.5) . The non-used capacity can be dynamically allocated to new generators. To host those new generators, while keeping a safe operation of the network (i.e. with current flows within the "dynamic" thermal limits), flexibility of DGs is then required. The deployment of an ANM system together with DLR sensors manages this flexibility: based on the measurement of the current flow and on the sag reserve on critical span owing to DLR, the ANM system takes control actions i.e. curtailment of the injection to maintain the network within normal operating limits.

\section{SIMULATION RESULTS}

A first evaluation has been done by Smarter Grid Solutions Ltd. (SGS) and shows the potential increase of energy export for all substations of the East loop if we consider the use of dynamic thermal rating on the most limited circuit. In these simulations a very conservative approach has been used.

Normal seasonal ratings are applied to all other circuits. This simulated DLR used by SGS is simplified compared to the Ampacimon one (detailed in other parts of this paper). DLR rating is based on wind speed data, assuming that the wind is always parallel to the line (conservative).

Another constraint for the future flexible generators (GFlex) of the East loop is that they can produce their rated power in normal network conditions, but they may be subject to curtailment during congestion constrained situations.

Using this simplified DLR hypothesis, results show that the total increase of produced energy would be $11,6 \%$ bigger with DLR on the most limiting circuit than witout DLR.

Current DLR sensors like Ampacimon could increase such gain up to $50 \%$ and over, as shown in Fig. 3 and 4. This is why a DLR development strategy has been chosen.

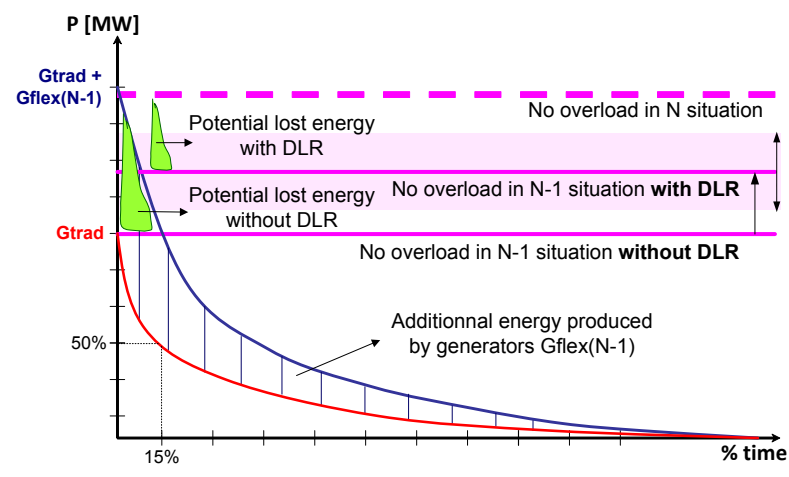

Fig. 5 : Potential benefit of DLR on produced energy by a wind farm. Gtrad (see also Fig. 6) is the basic wind farm generation with no possibility of curtailment (bottom curve). Gflex(N-1) is the part of the wind farm which can be curtailed (upper curves, with potential curtailment with or without DLR)

\section{FIRST RESULTS}

As described in the first part of this paper the impact on the production of renewable energy is substantial to the point that the connection of additional DG on the chosen network would have been impossible without the proposed operational approach (combining DLR and flexible wind production), but at no time this may affect the security of supply. In this regard several aspects are crucial: Reliable communication, safe fallback modes and Real-time measurement of the different components that affect network security.

\section{Curtailment tests}

Tests of curtailment have been performed on a new wind farm connected to the DSO $15 \mathrm{kV}$ network that is linked to the TSO $70 \mathrm{kV}$ "east loop" network.

According to the contractual arrangements, the wind farm can only be curtailed in N-1 situation.

In case of confirmed line overload detection, Elia generates a setpoint giving the maximal active power the generator can inject into the network.

This setpoint is then sent to the dispatching of the DSO ORES; it is forwarded to the ORES RTU installed in the client substation through a private and dedicated communication line. The setpoint is finally locally transferred by the ORES RTU to the generator automation systems where it is processed to be applied. The total active power injected onto the network by the generator must be reduced to the value of the setpoint within 15 minutes starting at the time of the line overload detection. 


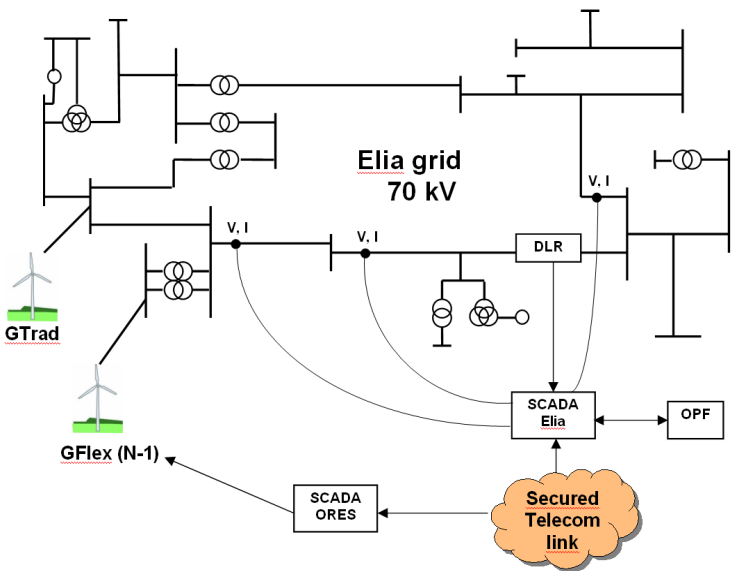

Fig. 6 : $70 \mathrm{kV}$ "East loop" in Belgium, equipped with several wind farms, Gtrad (no possible curtailment) and Gflex. The major limiting asset is one power line that will be equipped with DLR sensors. All communications links interfaced with TSO and DSO.

If the setpoint is not respected, ORES directly opens the breaker of the connection linking the generator to the grid in the HV/MV substation. This is to guarantee network security.

In case of failure of any communication link, the backup procedure is also forced and the connection is opened once again to avoid grid reliability problems.

Fig. 7 presents the evolution of the active power injected by the generator in response to several setpoints sent during the test phase. The first results show that power reduction can easily be reached within the allowed delay with the deployed modulation infrastructure and with this model of wind turbines (ENERCON E-82).

Constraints are located on the TSO $70 \mathrm{kV}$ lines in this test case and setpoints are generated by Elia and forwarded by ORES. Nevertheless the DSO could also initiate setpoints on his own.

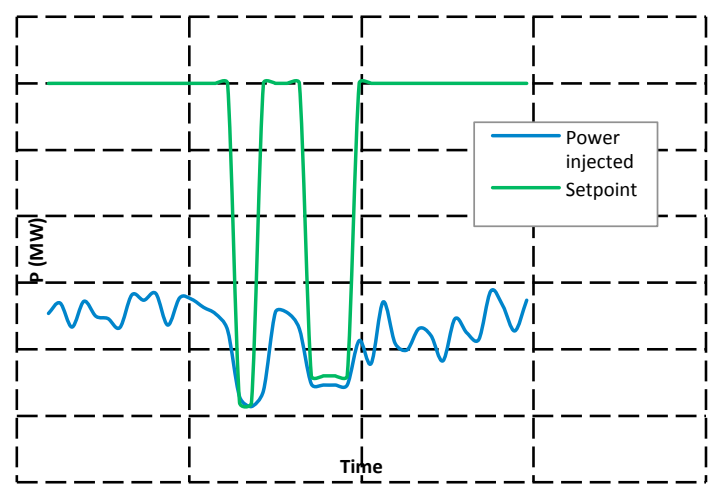

Fig. 7 : Results of modulation tests performed on a $10 \mathrm{MW}$ (rated power) windfarm (CORES) A $25 \mathrm{MW}$ setpoint means that power limitation is released.

\section{CONCLUSIONS AND FUTURE OUTLOOK}

Q1: What is the influence of a system of active network management using DLR on the investment strategy?

Answer : The proposed approach is probably not the solution for all the congestion issues related to the integration of renewable and/or distributed energy sources but it can certainly offer a solution for (many) areas. It can reduce the investment cost related to an increase of the connection capacity, required to host more distributed generation and speed up the connection process due to the significantly shorter implementation time then traditional means of uprating.

Q2: How is the reliability of the distribution system guaranteed in this new much more dynamic operation approach?

Answer : Increased visibility through monitoring and reliable, automated \& integrated control systems allow safe and reliable operation of the energy system. This is confirmed by our first tests in the field.

Further tests on farms with other types of wind turbines and for longer periods are required to fully qualify the proposed approach. The infrastructure deployed for the test case described in the current paper also allows sending modulation setpoints for the generator reactive power. This also will be investigated in the following phases of this project.

Dynamic Line Rating based on weather stations only have no significant impact because of the too conservative rules required. Only sensors installed directly on the power lines may give access to actual (certified) dynamic ratings (in both real time and short time forecast as needed for wind farms). 50\% more power installed and more may be gained in some locations if flexibility of (some of) the wind turbines (or others types of DGs) is accepted. 


\section{ACKNOWLEDGMENTS}

The authors wish to acknowledge the input of Smarter Grid solutions (CEO Robert Currie), ltd. (UK) in this project. Their previous experience see [3] in this field was instrumental for the overall success of the project. The case study on Brugge-Slijkens detailed in this paper has been supported by European project TWENTIES (2010-2013), more detailed on www.twenties-project.eu

\section{REFERENCES}

[1] Cloet, E., Lilien J.L. Uprating Transmission Lines through the use of an innovative real-time monitoring system. Proceedings of the IEEE 12th International Conference \& Exposition on Transmission \& Distribution. ESMO 2011 • May 16-19, 2011 • Providence, Rhode Island. http://hdl.handle.net/2268/102185

[2] O. Durieux, V. De Wilde, J-J. Lambin, S. Otjacques, M. Lefort, 2011, "Smart Grid Technologies Feasibility Study: Increasing decentralized generation power injection using global active network management" in Proc. 2011 CIRED (Frankfort, Germany, June 2011)

[3] Currie, R. A. F., Ault, G. W., Foote, C. E. T., McNeil, N. and Gooding, A. K.; "Smarter Ways to Provide Grid Connections for Renewable Generators"; IEEE PES General Meeting, Minneapolis, USA, July, 2010 